\title{
JULIO C. TELLO, SÍMBOLO DE PERUANIDAD
}

\author{
Toribio Mejía Xesspe
}

Si la voz "peruanidad" significa la esencia material y espiritual de nuestra nacionalidad, hay que convenir que ella tiene raíces profundas en nuestra Patria desde tiempos remotos, porque no otra cosa revela el origen y proeza de Ayar Manko, uno de los cuatro hermanos legendarios, que salió de la Cueva de Tampu-toqo, con la misión de ser, primero, cazador y recolector de los Andes peruanos; después, ser el gran domesticador de las plantas y animales silvestres; luego, convertirse en el hombre inteligente y organizador del ayllus y llactas, es decir aldeas y pueblos, para culminar, finamente, en el Héroe civilizador y creador del Imperio de los Inkas.

En los tiempos actuales, Julio C. Tello viene a ser el responsable genuino de la estirpe de los hermanos Ayar, porque su vida y obra tienden a demostrar el valor humano que heredó de sus antepasados; sólo así se explica el interés que tuvo para desempolvar el archivo milenario de pueblos y naciones fenecidos como Chavín, Paracas y otros de ser el portaestandarte de la reivindicación de sus hermanos de sangre y alma, cuando levantó su voz de protesta en 1921, desde el escaño parlamentario de la Cámara de Diputados, como representante de su provincia, Huarochirí.

Estos antecedentes históricos nos obligan a considerar la personalidad de Julio C. Tello, como símbolo de peruanidad. Para justificar este calificativo es necesario recordar algunos pasajes sobresalientes de su vida, ya que de este modo podemos aquilatar el justo valor de su espíritu nacionalista y científico.

He aquí ciertos datos sobre su origen andino y sobre el resultado de sus actividades como maestro universitario y como arqueólogo defensor del patrimonio nacional.

En los días trágicos de la Historia Nacional, en circunstancias que retumbaban los cañonazos del Monitor Huáscar en las aguas del Pacífico y en momentos de conmemoración social que afligía al pueblo peruano, con motivo de la guerra del 79, allá, en la anfractuosidad cisandina de Anan Yauyo, al pie del nevado Pariaqaqa, palpitaba un ser en las entrañas de una india joven y robusta llamada María Asunción Rojas, esposa de Julián Tello García, ambos naturales y vecinos de la Villa de Huarochirí. Pocos meses después, el 11 de abril de 1880, surgía a la vida humana el niño sharuko, llamado posteriormente Julio César Tello Rojas. Fue así como vino al mundo esta criatura andina, dotada con energías físicas y mentales extraordinarias para honrar la memoria de sus mayores, para dignificar el espíritu de su estirpe y para dar gloria a su patria. 
Como el recuerdo de sus antepasados, que fue trasmitido, oralmente sobre las hazañas de los héroes huarochiranos, como los Nina Willka, Yaqsha Willka, Kasha-waringa y otros, que secundaron la sublevación de Manko II en 1536; la rebelión de los kurakas o caciques de Huarochirí y Canta contra los abusos de los Corregidores, en 1750 cuyo documento de protesta ante el Rey de España, bajo el rubro de "Exclamación Reinvindicacionista de 1750", fue llevado clandestinamente a Madrid por el lego franciscano Calixto de San José Tupac Inca, natural de Tarma y descendiente de Tupak Inka Yupanqui, según los testimonios publicados recientemente en la revista de Historia y Cultura del Museo Nacional de Historia, № 3, Lima, 1969; el niño sharuko fue alimentado -como el mismo lo dijo más tarde-con la savia indígena, con las que se alimentaron en tiempos prehispánicos, los gigantes que construyeron los templos y palacios de Chavín, Tiahuanaco, Cusco y otros centros importantes del Antiguo Perú. Así creció Sharuko durante su niñez en la pintoresca campiña de Huarochirí, nutriendo su espíritu con las leyendas de Koniraya, Choqesuso, Watiakuri y otros personajes mitológicos de aquella región.

El despegue que inició Sharuko en 1893, hacia Lima, a la edad de trece años, fue providencial, porque le sirvió para conectarse con otros hombres, para recibir la influencia de la civilización europea y para escalar, peldaño tras peldaño, hacia la cumbre de la fama, fue así como traspasó los umbrales de colegios y universidades, cosechó lauros y distinciones en congresos científicos nacionales e internacionales, removió los cimientos vetustos de nuestras instituciones y organizó museos nacionales para el conocimiento de la historia patria.

Sirvan de ejemplo algunos triunfos obtenido en el país y el extranjero, tales como: la beca del Estado por tres años en las Universidades de Harvard, Berlín, Londres y París, tras su graduación de Médico y Cirujano de la Facultad de Medicina de Lima, en 1909; el grado académico de Master of Arts en la Universidad de Harvard, en 1911; la exposición brillante de su trabajo Prehistoric Threpaning among the Yauyos of Peru en el XVIII Congreso Internacional de Americanistas de Londres, en 1912; el triunfo político como Diputado Nacional por la Provincia de Huarochirí, en 1914, ante su contendor de campanillas y protegido del notable político don Ricardo Bentín.

Estas ocurrencias fueron las que facilitaron a Julio C. Tello el desenvolvimiento favorable de sus planes, en pro de las investigaciones antropológicas. Particularmente arqueológicas de diferentes regiones del país. El resultado de estas actividades se halla registrado en libretas manuscritas con ilustraciones de mapas, dibujos y fotografías, así como en estudios monográficos, en su mayoría inéditos; y el fruto de sus investigaciones arqueológicas se encuentra depositado en los Museos del Estado y de la Universidad Nacional Mayor de San Marcos. Llegará el día en que se conozca mejor la obra material e intelectual de Tello, cuando se exhiban todas las colecciones arqueológicas y cuando se publiquen sus trabajos inéditos.

Pasando a otro tema diremos: ¿Qué hizo Julio C. Tello a favor de la educación nacional? La respuesta es, sencillamente, muy sugestiva porque trató el problema desde el ángulo nacionalista y objetivo, tras una experiencia adquirida en institutos y universidades norteamericanas y europeas durante el periodo becario.

En primer lugar enfocó la Reforma Universitaria de 1921, en su calidad de miembro de la Comisión de Educación de la Cámara de Diputados, en colaboración con el maestro y diputado, José Antonio Encinas, con visión futurista y aplicable a la sociedad democrática del Perú. En efecto, con el propósito de modificar la Constitución Universitaria de 1920 cuyos principios fueron los siguientes:

1. Considerar a la Universidad Peruana como institución docente de altos estudios e investigaciones científicas, de modo que su función esencial sea enseñar todo conocimiento arreglado a sistemas pedagógicos.

2. Conservar los conocimientos y experiencias adquiridas por la civilización, mediante bibliotecas y museos que archiven e ilustren la historia del saber, de la vida y de la naturaleza. 
3. Investigar, esto es, procurar descubrir constantemente nuevas verdades, a fin de extender los límites del conocimiento presente, orientando sus actividades de preferencia hacia la solución de los problemas nacionales.

4. Propagar los conocimientos a las masas populares, mediante la colaboración de la Federación de Estudiantes, cuyos maestros sean los propios estudiantes, que desempeñen la docencia en forma gratuita.

En el curso de la exposición y defensa del Proyecto en referencia, que se llevó a cabo en las secciones de 5 y 6 de diciembre de 1921, Tello trató los puntos siguientes: Carácter nacionalista del Proyecto; La civilización es una y no es privilegio de una raza determinada; fuentes utilizadas en la confección del Proyecto; Junta de Supervigilancia y la Autonomía de la Universidad; La Biblioteca Nacional debe ser confederada a la Universidad; el principio de cooperación y coordinación en la nueva organización Universitaria; deberes profesionales; la Universidad y el Museo Nacional; La Sociedad Geográfica y la Universidad; La Facultad de Ciencias y la Facultad de Teología.

\section{Al finalizar su exposición dijo:}

"Este proyecto tiene, como las universidades americanas, doble finalidad: idealista y utilitaria. Está escrito con un criterio en apariencia doble, pero en realidad es uno solo, porque lo útil y práctico es el complemento de lo ideal. No hay una sola palabra, un solo pensamiento que pueda ser calificado como utópico e irrealizable. Este proyecto refleja algunas de las ideas democráticas del presente siglo; abre las puertas de la enseñanza de par en par; rompe las ligaduras y destruye los obstáculos; se empeña en amalgamar los diferentes elementos étnicos de la nacionalidad y derrumba esas capas artificiales que sobreviven desde los tiempos de la dominación española.

No es este proyecto para que rija en Inglaterra o Estados Unidos de Norte América. No. Es sólo para el Perú y para el indígena -ese infeliz que baja a Lima sólo para darse cuenta de su incapacidad y vuelve a su tierra a ser alcohólico o tinterillo- para que adquiera un ideal que perseguir, una preparación adecuada que le haga enérgico, firme y resuelto para lograr sus propósitos.

Si algún día imperara esta Constitución Universitaria, ya no tendrá el país sólo esos grandes nombres sonoros de personalidades que nos han dado gloria, como los García Calderón, los Villarán, los Riva Agüero y otros tantos, sino que habrían nombres más modestos, como Choquehuanca, Qespe, Waman, Condori, Madani, Asto-Poma, Chukillanki, Chamo-Chumbi, manko, Chipana, Huapaya, etc. que hoy llevan los peruanos que no han tenido oportunidad, como la tuvieron aquellos, para seguir en su propio país, porque las instituciones educadoras no fueron para ellos, de ahí que no han podido hasta ahora divisar la meta de sus aspiraciones; apenas, si algunos sacados por casualidad del terruño ha ido a los Estados Unidos a traernos nuevas ideas democráticas.

Ha llegado la oportunidad para que este Parlamento, compuesto por demócratas ilustres sancione esta Ley. Ha llegado el momento para que los hombres que tienen abundante sangre indígena en sus venas apoyen este Proyecto, porque él va a servir para redimir a nuestros hermanos, para favorecer a los cuatro millones de indígenas, que llevan una vida miserable en las alturas, para ofrecer igual oportunidad a los que tienen sangre española, inglesa o india, a fin de que todos unidos, bajo un mismo propósito y un mismo ideal, busquemos la grandeza de la Patria".

Una voz enérgica salida de la barra o galería de la Cámara de Diputados diciendo "Bravo Tello", "Viva el Indio", provocó una ovación prolongada en todo el recinto, con lo que concluyó la intervención del Diputado por Huarochirí. Este acontecimiento histórico a favor de la Educación Nacional ocurrió en las sesiones de la Cámara durante los días 5 y 6 de diciembre de 1921 y está registrado en el Diario de Debates del citado año.

Por otro lado iqué hizo Tello a favor de la instrucción popular de los ciudadanos que vi- 
ven fuera de la capital de la República o de las capitales Departamentales y Provinciales? Siendo testigo de la ignorancia en que viven los habitantes de las aldeas y pueblos de la sierra y siendo candidato a la diputación de su provincia, en 1917, fundó un Seminario titulado Evolución con el propósito de despertar el interés de las gentes hacia los conocimientos elementales de geografía, sociología e historia del país. Con el seudónimo de Gossip -palabra inglesa que significa murmuración, chisme- escribió una serie de artículos sobre moral y enseñanza tecnológica, sobre el vicio al alcohol y de la coca, sobre la rehabilitación del espíritu nacionalista y sobre otros temas de interés regional. En el primer número de Evolución escribió lo siguiente:

"La provincia de Huarochirí es una parte de la Patria que, geográfica y socialmente, se halla unificada suficientemente como para poseer verdadera conciencia de su propia unidad, como para sentir claro su diferencia de las otras provincias del Perú. Por eso iniciamos una campaña hoy contra todo rezago de apocamiento, de hipocresía, de desconfianza y esclavitud los que son degeneraciones del carácter que hemos heredado de nuestros antecesores indígenas, adquiridas bajo el pesado yugo de la ignorancia y de la tiranía española. Nos consideramos ya un poco más arriba del nivel de las llamas, que viven felices y unidas bajo el cuidado de un pastor; por eso ambicionamos ser hombres educados, altivos, enérgicos e independientes, no masas inertes, sujetas a la voluntad del primer mercante, que invoca la unión del rebaño en provecho propio."

Para conseguir que la instrucción primaria y secundaria tuviera resultados favorables en el desarrollo cultural de los pueblos, propició, en 1926, la creación de una Sección de Educación en la Facultad de Ciencias de la Universidad Nacional Mayor de San Marcos, mediante un dictamen presentado al Consejo de Facultades en el que planteó cuatro pun-

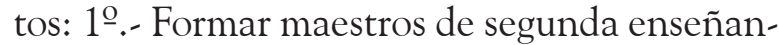
za, de escuelas vocacionales e inspectores de instrucción primaria; 2‥-Ofrecer preparación técnica para desempeñar los cargos de Director de Colegio, Director y Maestro de Escuela
Normal y Profesor de la propia sección de educación; 3‥- Ofrecer oportunidad al maestro de escuela elemental en ejercicio para estudiar su profesión bajo dirección técnica; y 4ํ‥-Fomentar los Seminarios de Educación, orientándolos hacia las investigaciones relacionadas con los problemas educacionales del país.

Asimismo, con otro dictamen de la Comisión de Instrucciones de la Cámara de Diputados, presentado en Noviembre de 1927, apoyó el proyecto del Ejecutivo para la previsión de becas en el extranjero, a favor de profesionales graduados o diplomados en las instituciones de enseñanza superior y escuelas normales, con la finalidad de asegurar la formación de especialistas en determinados ramos del conocimiento. Al respecto dijo: "la creación de unos treinta pensionados en el extranjero tiende, aunque en modesta escala, a satisfacer una exigencia apremiante de las aspiraciones culturales del país. Es obvio afirmar que uno de los medios más eficaces de propender al progreso intelectual de una nación es atrayendo hacia ella, las ideas y experiencias de los centros de mayor cultura. Los países relativamente nuevos que hoy figuran como grandes potencias en el mundo civilizado, como los Estados Unidos de Norte América y el Japón, deben el florecimiento de sus instituciones científicas, técnicas y educacionales a esta política de intercambio intelectual". Hasta aquí sus palabras.

Por último, en el discurso que pronunció en la ceremonia de inauguración del Museo de Arqueología Peruana el 13 de diciembre de 1924, al referirse a las funciones educacionales del Museo dijo: "El Museo es una institución educacional democrática por excelencia; el medio más eficaz para vulgarizar las enseñanzas de la historia. Si tenemos conciencia de nuestros deberes para con el destino de la Patria, estamos obligados a trabajar empeñosamente en la magna labor de educar al pueblo, despertando el espíritu solidario del grupo y forjando así la conciencia nacional. Bien sabemos que el cataclismo sorpresivo de la conquista paralizó las actividades creadoras de los indígenas; destruyó, junto con su civilización, los fundamentos de la nacionalidad. El lema político de los Inkas era trabajo especializado y organizado cooperativamente, base de todo progreso físico, intelectual y social. En los tiempos he- 
roicos del Imperio, todo estuvo encaminado a convertir al hombre, desde niño, en un factor dinámico, orientado hacia el dominio de la naturaleza, hacia la explotación de las riquezas del suelo, mediante el trabajo cooperativo intenso y tenaz. El trabajo fue un placer y no un dolor. Los que trabajan bajo la férula y amenaza mortal de un amo con poderes divinos y absolutos, esos no se especializan en las artes o en las industrias, que demandan el ejercicio de facultades mentales y emotivas superiores. No habrían jamás producido aquellas obras que revelan labor paciente, hasta convertirse en ocupación placentera; trabajo corporativo organizado, he aquí lo que produjo la asombrosa civilización de ayer. Si las leyes biológicas han de cumplirse una vez más en la sociedad peruana, esto es lo que producirá la grandeza del porvenir."

Otro aspecto valioso de Julio C. Tello en pro de la prehistoria nacional fue condensado en tres grandes capítulos: museo, descubrimientos arqueológicos y defensa del patrimonio cultural.

A manera de síntesis debemos referirnos a estos temas, por cuanto ya se han divulgado en ocasiones anteriores, ora en libros y monografías, ora en artículos de prensa diaria y revistas nacionales, ora en charlas y conferencias, etc.

Museos.- Aunque este tipo de instituciones fue patrocinado desde los albores de la Emancipación política como la creación del Primer Museo nacional de Lima, mediante el Decreto Supremo de 20 de Abril de 1822 y su conversión en Museo Natural por Decreto Supremo de 3 de Junio de 1836, no dejó de ser más que simple depósito de colecciones muy reducidas en calidad y cantidad, hasta que por Decreto de 17 de Mayo de 1872 fue involucrado a la Sociedad de Bellas Artes, que funcionaba en una Sección del Palacio de la Exposición, donde, finalmente dicho Museo sufrió menoscabo y pillaje durante la ocupación de Lima por la soldadesca chilena, en 1881. Veinticinco años después de este vandalismo se fundó y organizó un auténtico museo con el rubro de "Museo de Historia Nacional", bajo dependencia del Instituto Histórico del Perú, a mérito del Decreto Supremo de 6 de Mayo de 1905, siendo inau- gurado solemnemente el 29 de Julio de 1906, bajo la dirección técnica y administrativa del arqueólogo alemán, Max Uhle. Sin embargo, esta flamante institución que comprendía dos secciones: una histórica y otra arqueológica, no prosperó como debiera ser debido a la ausencia del arqueólogo-director por finiquitación de su contrato, en 1911 y por otras causas de carácter administrativo, hasta que en 1935, las colecciones históricas y arqueológicas pasan a incrementar los fondos culturales de otras instituciones del Estado.

Mientras ocurrían estos hechos surge la personalidad de Julio C. Tello como arqueólogo y como fundador de museos. Así, en 1919, funda el Museo de Arqueología y Etnología de la Universidad Nacional Mayor de San Marcos, a base de las colecciones reunidas en la Expedición Arqueológica al Departamento de Ancash; en 1924, fomentó la adquisición de las colecciones del Museo particular "Víctor Larco Herrera" para organizar, por cuenta del gobierno, el Museo de Arqueología Peruana, cuya dirección asumió con carácter de ad-honorem durante 5 años; en 1936 organizó el Museo de Antropología de Pueblo Libre, a base de los materiales obtenidos en campañas arqueológicas de los años 1925-1930, bajo los auspicios del Instituto de Investigaciones Antropológicas de la Universidad Nacional Mayor de San Marcos y el Museo Nacional; y en 1945, promovió la reorganización de los museos estatales y consiguió la fundación del Museo Nacional de Antropología y Arqueología. Además, fundó en 1940, el primer Museo de Sitio en las ruinas del Templo de Chavín de Huántar con 119 piezas líticas del arte escultórico, perteneciente a dicho templo, que anteriormente estuvieron en corrales y potreros de los vecinos del pueblo, sirviendo como estacas para amarrar bestias, como materiales de construcción y de adorno en casas particulares y plazas o bien como batanes y otros usos domésticos en los hogares campesinos. Fatalmente este museo de Chavín desapareció con todos sus materiales en el desastre aluviónico de 17 de enero de 1945. No obstante esta desgracia, la previsión de Tello pudo salvar el recuerdo de estas esculturas desaparecidas, mediante los moldes sacados en yeso por Luis Ccosi durante las excavaciones de noviembre y diciembre de 1940, moldes que posterior- 
mente fueron reproducidos en Lima para su exhibición en el Museo Nacional de Antropología y Arqueología.

Descubrimientos arqueológicos. Estos se realizaron con doble finalidad: 1) para establecer asociaciones entre los millares de objetos selectos, que fueron extraídos por huaqueros con fines meramente comerciales sin ningún dato histórico de sus hallazgos; y aquellos objetos que han sido extraídos mediante procedimientos técnicos con fines de estudio científico; 2) para incrementar las colecciones y determinar el origen y desarrollo de las civilizaciones andinas. Con estos propósitos, Tello organizó un plan de exploraciones y excavaciones con el auxilio de especialistas y estudiantes peruanos y extranjeros, y el soporte económico de entidades estatales y de instituciones científicas norteamericanas. En esta forma realizó los siguientes trabajos de campo:

- 1915: Reconocimiento arqueológico de los departamentos de Ica, Arequipa, Puno y Cusco, inclusive Tiahuanaco en Bolivia.

- 1916: Exploración arqueológica y etnológica en las provincias de Ayabaca, Huancabamba y Jaén en el norte peruano, como integrante de la Expedición Científica de la Universidad de harvard.

- 1925: a) Excavaciones de la Waca Malena en el Valle de Asia, provincia de Cañete, durante tres meses, por cuenta del Museo de Arqueología de San Marcos; b) exploraciones en el valle de Cañete y Waka Cerro del Oro, en colaboración con el profesor Alfred Kroeber, de la Universidad de California; c) exploraciones en los valles de Chincha y Pisco, en compañía del profesor Samuel K. Lathrop de la Universidad de Harvard, cuyas actividades culminaron con el descubrimiento de Paracas en la península del mismo nombre.

- 1927: Expedición arqueológica al Departamento de Ica, durante once meses, para estudiar y excavar las tumbas en los valles de Nasca, Kopara o Las Trancas, El Ingenio, Wayurí y para proseguir las excavaciones en los cementerios de Cerro Colorado en Paracas, que culminó con el descubrimien- to de las Grandes Necrópolis y sus fardos funerarios.

- 1931: Exploraciones arqueológicas en la cuenca del río Mantaro y sus afluentes y reconocimiento de las ruinas de WariWakaurara y Qoncho-pata en los alrededores de la ciudad de Ayacucho.

- 1933: Exploración arqueológica del valle de Nepeña, y descubrimiento de los templos chavinoides de Cerro Blanco y Punkurí, cuyos trabajos de exploración y estudio se llevaron a cabo hasta mediados de 1934.

- 1935: Exploración del Alto Huallaga y descubrimiento del templo Pre-Chavín de Kotosh, en los alrededores de la ciudad de Huanuco.

- 1935: Exploración en las ruinas de Arequipa, Puno y Cusco en compañía de Luis E. Valcárcel y descubrimiento del yacimiento de Pucara, provincia de Lampa, con restos de cerámica chavinoide o Pre-Tiahuanaco.

- 1937: Expedición arqueológica al Marañón, bajo los auspicios de la Universidad de San Marcos y apoyo económico de Nelson A. Rockefeller. Durante esta expedición se descubrieron los monumentos de Pallka, Moxeke y Cerro Sechín en el valle de Casma; el acueducto megalítico de Kumbemayo, en Cajamarca; las ruinas de Chokta, en Celendín; los mausoleos megalíticos y hemisferios de Yanakancha en Hualgayoc y las ruinas del templo de Nuna-marka, en Chilia, provincia de Pataz.

- 1940: Exploraciones y excavaciones arqueológicas en las ruinas de Chavín de Huántar y sus alrededores, bajo los auspicios de la Dirección del Ministerio de Fomento.

- 1940: Exploraciones y excavaciones en las ruinas del Templo de Pachacamac, con el soporte económico de la Junta Pro-desocupados Departamental de Lima. Los trabajos se efectuaron hasta 1946, durante los cuales se descubrieron las cisternas en el Templo de la Luna o Mamacona y los 
restos de construcciones inka de piedras labradas, debajo de las estructuras inka de adobes rectangulares. En este último periodo se iniciaron los trabajos de restauración de los compartimientos del referido Templo de la Luna.

- 1942: Expedición Arqueológica a la Hoya del Urubamba, Cusco, durante seis meses, bajo los auspicios de Viking Fund de Nueva York, para continuar las exploraciones de Wenner-Gren y Paul Fejos. En esta ocasión se hicieron exploraciones y reconocimientos de ruinas a lo largo de los ríos Mantaro, Apurímac y Urubamba, culminando con el descubrimiento de la ciudadela de Wiñay Waina, cerca de Machu Picchu.

- 1945: Excavaciones arqueológicas en las ruinas de Ancón por encargo del Patronato Nacional de Arqueología, y por cuenta de la Compañía Urbanizadora Miramar.

- 1946: Exploraciones y excavaciones en el templo de Kuntur Wasi (La Capa) en los alrededores del pueblo de San Pablo, Provincia de Cajamarca.

Además se practicaron otros reconocimientos arqueológicos bajo la dirección de Tello, como en Laraos, provincia de Yauyos, en 1934; en Illimo, provincia de Lambayeque, 1937; en Huaitará y alrededores, en 1941; en Ocoña, Andaray, Chuquibamba y Majes, en 1943; en la Cuenca del río Pampas y río Apurimac, incluyendo Choque-Kirao y Viticos, en 1944.

Defensa del Patrimonio Cultural.- Esta labor proteccionista comprende dos funciones de gran responsabilidad: conservación de los monumentos prehispánicos, que existen por doquier del territorio nacional, como testimonios objetivos de las civilizaciones pretéritas; y conservación de la colecciones arqueológicas que atesoran y exhiben los museos nacionales, como reliquias de industria, arte y ciencia de los primitivos pobladores del Perú.

Para lo primero Tello se interesó, desde su asiento en la Cámara de Diputados, para conseguir la dación de la Ley n⿳⺈ 6634, de 13 de junio de 1929. Comprendía que sin la ley debidamente sancionada y aprobada por el Parlamento Nacional, era imposible detener la destrucción vandálica de los monumentos arqueológicos, porque simples decretos y resoluciones del gobierno eran burlados impunemente por los traficantes de antigüedades, que exportaban frecuentemente para satisfacer la demanda de los museos extranjeros. Por esta razón Tello colaboró en la redacción y aprobación de la mencionada ley 6634, cuyo articulado protege los monumentos de origen prehispánico; reconoce los derechos de tenencia de los coleccionistas de objetos adquiridos antes de la dación de la ley, da facilidades para estudios arqueológicos a los especialistas peruanos y extranjeros, así como a los representantes de instituciones científicas que tengan interés en realizar exploraciones y excavaciones, previo permiso del Patronato Nacional de Arqueología y autorización gubernativa. Gracias a esta ley se pudo amenguar el saqueo de tumbas y exportación de colecciones, mientras vivía Tello, gran defensor del patrimonio arqueológico, porque él, personalmente, intervenía en la aplicación de dicha ley.

Para lo segundo, esto es, la conservación de las colecciones que poseen los museos del Estado, Tello, en su condición del Director del Museo Arqueológico, hizo gestiones ante el gobierno y personas amantes del pasado peruano, para construir un local adecuado que sirviera a la vez de laboratorio para investigaciones científicas y artísticas y de presentación digna de las maravillosas obras de arte de nuestros antepasados. Con esta finalidad consiguió en 1941 la donación d un terreno, contiguo al local del actual Museo Nacional de Antropología y Arqueología, de propiedad de Don Germán Luna Iglesias, para levantar un edificio de dos pisos. Para la financiación de esta obra gestionó la creación de un pequeño impuesto de $1 \%$ sobre el importe de los gastos de alojamiento en los hoteles de la República, Ley no 10167 de 15 de junio de 1945. Pero la muerte prematura de Tello frustró la realización de dicha obra, pues por otra ley del a Junta de Gobierno la no 11418 de 7 de julio de 1950, los fondos recaudados por la ley $\mathrm{n}^{\mathrm{o}} 10167$, fueron transferidos a la cuenta llamada "Fondo de Educación Nacional". Es por esta causa que el Museo Nacional de Antropología y Arqueología carece hasta hoy un local de su categoría y en el que funciona 
desde hace más de 30 años es de madera, caña y barro que originariamente perteneció al Museo Bolivariano. Ojalá algún día este edificio vetusto e inapropiado sea sustituido por otro de mayor rango como lo ambicionaba Julio C. Tello.

Como si no fuera suficiente para el ideal de Tello, lo que acabamos de mencionar sobre el destino de Museo, él quiso ser testigo y guardián permanente de las reliquias de sus antecesores, porque él tomó parte en sus adquisiciones y descubiertos, como de aquellas manufacturas textiles que amortajaban el cuerpo de los sacerdotes de Paracas; de aquellos tazones ceremoniales de Nasca, restaurados pacientemente de millares de fragmentos; de aquellas piezas finas y pequeñas de cerámica que acompañaban a los muertos de las naciones desaparecidas como los Chanka, Nasca, Rukana, Chimú o Inka, decimos que quiso permanecer en el recinto del Museo, donde había pasado gran parte de su vida de arqueólogo. Con tal propósito dispuesto testamentariamente que sus restos mortales reposaran junto a las reliquias de sus antepasados. Así se hizo después de su muerte ocurrida en Lima el 3 de junio de 1947. Desde entonces los restos humanos del fundador de muesos arqueológicos y descubridor de varias culturas ignoradas, descansan bajo tierra, cubiertos simbólicamente con la tierra traída de los pueblos de su provincia, Huarochirí.

Como si esto también fuera poco para la ambición de Julio C. Tello, quiso, igualmente por disposición testamentaria, que su patrimonio cultural, compuesto por una biblioteca de índole antropológica y de su archivo documental y científico, pasara en calidad de donación perpetua a la Universidad Nacional Mayor de San Marcos. Fue así como sus herederos -esposa e hijos- entregaron a la Universidad el 14 de agosto de 1947, por ante el Juez de Primera Instancia de Lima, Dr. Ernesto Vivanco Mujica, lo siguiente:

7,662 unidades de libros, folletos y revistas.

486 paquetes debidamente clasificados, catalogados y numerados, que corresponden a monografías inéditas, a libretas manuscritas conteniendo descripción de tumbas, cementerios, monumentos, especimenes, historia de los fardos funerarios de Paracas, Nasca y muchos otros datos sobre exploraciones, expediciones y descubrimientos arqueológicos, acompañados de multitud de mapas, esquemas, dibujos y fotografías, con miras a ser publicados por la Universidad.

Los libros y folletos se encuentran en la Biblioteca Central de la Universidad; y los paquetes, lacrados y sellados bajo la supervigilancia del Museo de Arqueología y Etnología.

De este valioso legado cultural se han publicado 4 volúmenes: Arqueología del Valle de Casma, 1956; Paracas, I Parte, 1959; Chavín, Cultura Matriz de la Civilización Andina, 1960; Historia de los Museos Nacionales, 1822-1946, 1967.

He aquí, a grandes rasgos, la vida y obra de Julio C. Tello, símbolo de peruanidad. 